\title{
IncRNA NEAT1 promotes cell proliferation and invasion by regulating miR-365/RGS20 in oral squamous cell carcinoma
}

\author{
GANG HUANG $^{1}, \mathrm{XIN} \mathrm{HE}^{2}$ and XIN-LI WEI ${ }^{1}$ \\ Departments of ${ }^{1}$ Stomatology and ${ }^{2}$ Scientific Research and Medical Education Management, \\ General Hospital of Benxi Iron and Steel Co., Ltd., Benxi, Liaoning 111700, P.R. China
}

Received August 30, 2017; Accepted February 7, 2018

DOI: $10.3892 / o r .2018 .6283$

\begin{abstract}
Long non-coding RNAs (lncRNAs) have emerged as critical regulators of tumor progression. However, the function and mechanism of lncRNA NEAT1 in oral squamous cell carcinoma (OSCC) are unclear. In the present study, NEAT1 was significantly upregulated in OSCC cells and tissues. High expression of NEAT1 was correlated with advanced TNM stage and poor survival of patients. Using bioinformatics prediction and experimental analysis, we determined that NEAT1 could negatively regulate the expression of miR-365. The expression of miR-365 was decreased in OSCC tissues and inversely correlated with NEAT1 in tumors. Functionally, knockdown of NEAT1 significantly inhibited cell proliferation and invasion and induced cell cycle arrest at the G0/G1 phase and apoptosis, whereas inhibition of miR-365 abolished the suppressive effect of NEAT1 knockdown on cellular processes. RGS20, a direct target of miR-365, could reverse the tumor suppressive role of miR-365 mimic by enhancing cell viability and motility. Moreover, the protein levels of RGS20, cyclin D1, E-cadherin, N-cadherin and vimentin could be regulated by the NEAT1/miR-365 axis. NEAT1 silencing also inhibited tumor growth in vivo. Collectively, we revealed that the NEAT1/miR-365/RGS20 axis may be a novel mechanism or therapeutic strategy for OSCC treatment.
\end{abstract}

\section{Introduction}

Oral squamous cell carcinoma (OSCC) is the most prevalent type of head and neck cancer and it accounts for nearly $90 \%$ of all oral cancer cases (1). Despite improvement in OSCC treatment over the past years, the 5-year survival rate of OSCC patients has not significantly improved (2). Therefore, identifying effective biomarkers and therapeutic targets is essential

Correspondence to: Dr Gang Huang, Department of Stomatology, General Hospital of Benxi Iron and Steel Co., Ltd., 29 Renmin Road, Benxi, Liaoning 111700, P.R. China

E-mail: huanggang_hg@126.com

Key words: NEAT1, miR-365, RGS20, proliferation, epithelialmesenchymal transition to acknowledge the molecular mechanisms underlying the progression of OSCC.

Long non-coding RNAs (lncRNAs), a class of regulatory transcripts greater than 200 nucleotides without protein-coding function, play a critical role in tumorigenesis through multiple mechanisms, including interaction with microRNAs (miRNAs) or proteins $(3,4)$. miRNAs can post-transcriptionally regulate gene expression via binding to the 3 '-untranslated region (UTR) of mRNAs (5). Both dysregulated lncRNAs and miRNAs were reported to be closely related with tumor cellular processes, including proliferation, differentiation, invasion and apoptosis $(6,7)$. For example, NEAT1 was found to be upregulated in several types of cancers (8-11). NEAT1 contributed to cell growth and metastasis and acted as a competing endogenous RNA (ceRNA) for miR-377-3p in lung cancer (12). NEAT1 epigenetically suppressed miR-129-5p expression by promoting the miR-129 related CpG island methylation (13). In addition, several other miRNAs, including miR-335, miR-107 and miR-101, were demonstrated to interact with NEAT1 and involved in NEAT1 regulated tumor-related biological processes (14-16). However, the expression and function of NEAT1 in the development of OSCC remain unclear.

Among the dysregulated miRNAs, miR-365 was reported to be decreased in colon cancer, inhibited cell cycle progression and induced apoptosis (17). NKX2-1, a direct target of miR-365, attenuated the suppressive function of miR-365 on cell proliferation in lung cancer (18). In gastric cancer, activation of Akt decreased the expression of miR-365, consequently promoting cell growth by increasing the expression of cyclin D1 and CDC25A (19).

In the present study, we determined that the expression of NEAT1 was increased in OSCC and associated with tumor progression. Knockdown of NEAT1 inhibited cell proliferation and invasion and induced cell cycle arrest at the G0/G1 phase. Furthermore, we found that NEAT1 could act as a ceRNA of miR-365 and therefore regulate its target gene RGS20.

\section{Materials and methods}

Cell lines and clinical samples. OSCC cell lines (SCC-9, SCC-25,HN4, Tca-8113 and Cal-27) were obtained from the Cell Bank of the Chinese Academy of Sciences (Shanghai, China) and cultured in Dulbecco's modified Eagle's medium (DMEM; Invitrogen; Thermo Fisher Scientific, Inc., Carlsbad, CA, USA) 
supplemented with $10 \%$ fetal bovine serum (FBS; Invitrogen; Thermo Fisher Scientific, Inc.), and $100 \mu \mathrm{g} / \mathrm{ml}$ penicillin/streptomycin (BioLight, Shanghai, China). A human normal oral keratinocyte cell line (hNOK) was used as a control. All cells were incubated at $37^{\circ} \mathrm{C}$ in a humidified atmosphere with $5 \% \mathrm{CO}_{2}$.

Thirty OSCC tissues and their adjacent non-tumor tissues were obtained from patients at the Department of Stomatology, General Hospital of Benxi Iron and Steel Co., Ltd. (Benxi, China), between 2010 and 2012. The present study was approved by the Research Ethics Committee of the General Hospital of Benxi Iron and Steel Group Co., Ltd., and written informed consents from patients were signed before surgery. None of the patients had a prior history of cancern or had received radiochemotherapy before surgery. All tissues were immediately snap-frozen in liquid nitrogen and stored at $-80^{\circ} \mathrm{C}$ until use. The clinicopathological characteristics of patients were summarized in Table I.

Quantitative real-time PCR ( $P P C R)$. Total RNA was isolated using TRIzol reagent (Invitrogen; Thermo Fisher Scientific, Inc.). To assess NEAT1 and RGS20 expression, a High Capacity cDNA Reverse Transcription kit (Applied Biosystems; Thermo Fisher Scientific, Inc., Foster City, CA, USA) and SYBR Premix Ex Taq (Takara Biotechnology Co., Ltd., Dalian, China) were used for reverse transcription (RT) and qPCR, respectively. For the expression of miR-365, the TaqMan MicroRNA Reverse Transcription kit and the TaqMan Universal Master Mix II (both from Applied Biosystems; Thermo Fisher Scientific, Inc.) were used forRT and qPCR, respectively. The results for NEAT1 and RGS20 were normalized to the expression of ATCB and miR-365 to the expression of U6. The relative expression level of each gene was calculated and normalized using the $2^{-\Delta \Delta \mathrm{Ct}}$ method.

Plasmidand oligonucleotide. The small interfering RNAs (siRNAs) specifically targeting NEAT1 (si-NEAT1 sense, 5'-GAGGGAUGAGGGUGAAGAA-3' and antisense, 5'-UUCUUCACCCU CAUCCCUC-3') and the negative control siRNA (si-NC) were obtained from Guangzhou RiboBio Co., Ltd. (Guangzhou, China). For in vivo analysis, cells were transfected with $6 \mu \mathrm{g}$ of sh-NEAT1 or the empty lentiviral vector, cultured with DMEM containing 20\% FBS for $36 \mathrm{~h}$. Lentiviral particles were harvested and used for infection. The target sequence of sh-NEAT1 was as follows: 5'-GCCATCAGCTTTGAATAAATT-3'. The human NEAT1 genewasligatedintothepGCMV/MCS/RFP/Neovector(Shanghai Genepharma Co., Ltd., Shanghai, China) and stable cell lines were generated by selection with Geneticin ${ }^{\circledR}$ (G418; Invitrogen; Thermo Fisher Scientific, Inc.). The packaged lentiviruses were named sh-NEAT1 and the empty lentiviral vector (sh-Ctrl) was used as a control. The miR-365 mimic, miR-365 inhibitor, mimic negative control (mim-NC), inhibitor negative control (inh-NC) sequences were obtained from Shanghai Genepharma Co., Ltd. Cells were transfected using Lipofectamine 2000 (Invitrogen; Thermo Fisher Scientific, Inc.). To restore RGS20 expression, the Cal-27 cells were transfected with a pcDNA3.1-RGS20 plasmid (pcRGS20), which contained the coding sequences but lacked the 3'-UTR of RGS20. Cells transfected with the empty vector were used as a control and named pcDNA.
Cell proliferation analysis. Cells $\left(1.5 \times 10^{3} /\right.$ well $)$ were plated in 96-well culture plates and cell viability was assessed every $24 \mathrm{~h}$ after transfection. MTT $[5 \mathrm{mg} / \mathrm{ml}$ in phosphate-buffered saline (PBS); Sigma-Aldrich, St. Louis, MO, USA] was added to each well and the plates were incubated at $37^{\circ} \mathrm{C}$. After $4 \mathrm{~h}$, $150 \mu$ l dimethyl sulfoxide (DMSO) was added to each well. The absorbance was measured at $490 \mathrm{~nm}$ on a microplate reader (Molecular Devices, LLC, Sunnyvale, CA, USA).

Cell cycle and apoptosis analysis. At $48 \mathrm{~h}$ post-transfection, cells were harvested, washed with PBS, and fixed with $70 \%$ ethanol. Then the fixed cells were washed with PBS, centrifuged at $1,500 \mathrm{x} \mathrm{g}$ for $5 \mathrm{~min}$ and subsequently treated with RNase A $(0.1 \mathrm{mg} / \mathrm{ml})$ and propidium iodide (PI; $0.05 \mathrm{mg} / \mathrm{ml})$ at $37^{\circ} \mathrm{C}$ for $30 \mathrm{~min}$. The stained cells were analyzed by flow cytometry (FACSCalibur; BD Biosciences, San Jose, CA, USA). For the apoptosis assay, $48 \mathrm{~h}$ post-transfection, the cells were collected by trypsinization and washed twice with serum-containing medium. The cells were collected and resuspended in 1X Annexin V Binding buffer (Annexin V-FITC Apoptosis Detection kit; BD Pharmingen; BD Biosciences) at a concentration of $1 \times 10^{6}$ cells $/ \mathrm{ml}$. Then, $5 \mu \mathrm{l}$ of FITC Annexin $\mathrm{V}$ and $5 \mu \mathrm{l}$ PI (BD Pharmingen; BD Biosciences) were added to $100 \mu \mathrm{l}$ of the cell suspension. After incubation for $10 \mathrm{~min}$ at room temperature in the dark, $400 \mu \mathrm{l}$ of binding buffer was added. Apoptosis was analyzed by flow cytometry (FACSCalibur; BD Biosciences) and the data were analyzed using CellQuest software (BD Biosciences).

Cell invasion assay. Cell invasion abilities were detected using Transwell chambers precoated with Matrigel (BD Biosciences). DMEM with 10\% FBS was added to the lower chamber. OSCC cells were transfected, incubated, and then starved in serum-free DMEM overnight. Subsequently, they were resuspended $\left(1 \times 10^{5}\right.$ cells) in serum-free medium, which was added to the upper chamber. Twenty-four hours later, the cells that had invaded to the lower surface of the membrane were fixed, stained and counted under an inverted microscope (Olympus, Tokyo, Japan) by counting five random fields.

Luciferase activity assay. The fragment from NEAT1 containing the predicted miR-365 binding site was amplified by PCR and cloned into a pmirGLO Dual-Luciferase Target Vector (Promega Corp., Madison, WI, USA) to form the NEAT1-wild-type reporter vector (NEAT1-WT). The mutant was generated by mutating the miR-365 seed region binding site and named NEAT1-MUT. Cells were co-transfected with either wild-type fragments or mutant fragments and miR-365 mimic or mim-NC using Lipofectamine 2000. A luciferase reporter assay was performed using the Dual-Luciferase Reporter Assay system (Promega Corp.).

The 3'-UTR of RGS20 containing the putative binding sites for miR-365 was amplified by PCR and cloned into the pGL3-luciferase reporter plasmid (Promega Corp.). Mutations in the miR-365-binding site of RGS20 3'-UTR were generated by the QuikChange Site-Directed Mutagenesis kit (Stratagene, La Jolla, CA, USA). Cells were co-transfected with miR-365 mimic (or mim-NC) and the reported vector with the wild-type (WT) or mutant (MUT) 3'-UTR of RGS20. Luciferase activity was assessed after incubation for $48 \mathrm{~h}$ at $37^{\circ} \mathrm{C}$. 
Table I. The expression levels of NEAT1 and miR-365 in subgroups of OSCC cases.

\begin{tabular}{|c|c|c|c|c|c|}
\hline Characteristics & Cases, $n=30$ & NEAT1 levels & P-value & miR-365 levels & P-value \\
\hline Age (years) & & & $0.657^{\mathrm{a}}$ & & $0.966^{\mathrm{a}}$ \\
\hline$<55$ & 12 & $3.057 \pm 1.094$ & & $0.616 \pm 0.328$ & \\
\hline$\geq 55$ & 18 & $3.297 \pm 1.268$ & & $0.627 \pm 0.396$ & \\
\hline Sex & & & $0.094^{\mathrm{a}}$ & & $0.933^{\mathrm{a}}$ \\
\hline Female & 13 & $3.432 \pm 1.231$ & & $0.593 \pm 0.301$ & \\
\hline Male & 17 & $2.680 \pm 1.067$ & & $0.645 \pm 0.413$ & \\
\hline LNM status & & & $0.009^{\mathrm{a}}$ & & $0.244^{\mathrm{a}}$ \\
\hline Negative & 20 & $2.562 \pm 0.769$ & & $0.669 \pm 0.353$ & \\
\hline Positive & 10 & $3.892 \pm 1.394$ & & $0.529 \pm 0.385$ & \\
\hline TNM stage & & & $0.018^{a}$ & & $0.039^{a}$ \\
\hline I, II & 16 & $2.507 \pm 0.778$ & & $0.743 \pm 0.355$ & \\
\hline III, IV & 14 & $3.576 \pm 1.328$ & & $0.486 \pm 0.334$ & \\
\hline Smoking & & & $0.148^{\mathrm{a}}$ & & $0.258^{\mathrm{a}}$ \\
\hline Never & 9 & $2.445 \pm 0.809$ & & $0.761 \pm 0.419$ & \\
\hline Quit & 21 & $3.246 \pm 1.250$ & & $0.563 \pm 0.331$ & \\
\hline Differentiation & & & $0.603^{\mathrm{a}}$ & & $0.835^{\mathrm{a}}$ \\
\hline Well & 16 & $2.893 \pm 1.241$ & & $0.605 \pm 0.364$ & \\
\hline Moderate/poor & 14 & $3.135 \pm 1.144$ & & $0.643 \pm 0.377$ & \\
\hline Location & & & $0.443^{\mathrm{b}}$ & & $0.929^{b}$ \\
\hline Tongue & 19 & $2.987 \pm 1.361$ & & $0.644 \pm 0.425$ & \\
\hline Cheek & 8 & $3.234 \pm 0.837$ & & $0.574 \pm 0.212$ & \\
\hline Gingiva & 3 & $2.517 \pm 0.767$ & & $0.621 \pm 0.357$ & \\
\hline
\end{tabular}

${ }^{a}$ Mann-Whitney test; ${ }^{b}$ Kruskal-Wallis test; $\mathrm{P}<0.05$ is indicated in bold. OSCC, oral squamous cell carcinoma; LNM, lymph node metastasis; never, no smoking history or has stopped smoking for $>5$ years; quit, has stopped smoking for $<5$ years.

Western blot analysis. Cells were lysed in RIPA lysis buffer (Beyotime Institute of Biotechnology, Shanghai, China) and the protein concentration was measured using a BCA protein assay kit (Thermo Fisher Scientific, Inc., Rockford, IL, USA). Equal amounts of protein were isolated using SDS-PAGE and then transferred to a polyvinylidene fluoride (PVDF) membrane (EMD Millipore, Bedford, MA, USA). The membranes were blocked in 5\% non-fat milk/TBST and incubated with primary antibodies. The primary antibodies RGS20 (1:500; cat. no. ab191500), cyclin D1 (1:1,000; cat. no. ab134175), E-cadherin (1:2,000; cat. no. ab15148), $\mathrm{N}$-cadherin $(1: 2,000$; cat. no. ab18203), vimentin $(1: 2,000$; cat. no. ab24525) and $\beta$-actin $(1: 2,000$; cat. no. ab8227) were purchased from Abcam (Cambridge, MA, USA). Subsequently, the membranes were incubated with goat anti-rabbit secondary antibodies (1:2,000; cat. no. ab150077) and the proteins were detected with ECL reagents (Pierce; Thermo Fisher Scientific, Rockford, IL, USA).

In vivo tumor growth assay. All animal procedures were in line with the guidelines of the Laboratory Animal Centre and were approved by the Ethics Committee of the General Hospital of Benxi Iron and Steel Co., Ltd. Ten female athymic $\mathrm{BALB} / \mathrm{c}$ nude mice (4-5 weeks) were used. A total of $200 \mu \mathrm{l}$ of PBS containing $2 \times 10^{7} \mathrm{Cal}-27$ cells expressing sh-NEAT1 or sh-NC were injected subcutaneously to the flanks of each mouse ( $\mathrm{n}=5$ for each group). The tumor size was measured every 7 days and was calculated using the following formula: $0.5 \mathrm{x}$ length $\mathrm{x}$ width ${ }^{2}$. Four weeks later, the mice were sacrificed and the tumors were harvested.

Statistical analysis. SPSS 16.0 software (IBM, Armonk, NY, USA) and GraphPad Prism 5 software (GraphPad Software, Inc., San Diego, CA, USA) were used for statistical analysis. The paired samples t-test was used to compare gene expression levels between OSCC and non-tumor controls. The overall survival of patients was analyzed by the Kaplan-Meier method. One-way ANOVA or Student's t-test was used for comparisons between the groups. $\mathrm{P}<0.05$ was considered to indicate a statistically significant result.

\section{Results}

NEAT1 is overexpressed in OSCC cells and tissues. In order to know the relevance of NEAT1 in OSCC development, we assessed the endogenous levels of NEAT1 in OSCC cells. As shown in Fig. 1A, the expression of NEAT1 was significantly increased in OSCC cells compared to hNOK cells. Then, a qPCR assay was performed to evaluate the expression of NEAT1 in clinical samples. The expression of NEAT1 in OSCC tissues was significantly higher than that in matched non-tumor tissues ( $3.006 \pm 1.182$ vs. $1.712 \pm 0.971, \mathrm{P}=0.0004$; Fig. $1 \mathrm{~B})$. 
A

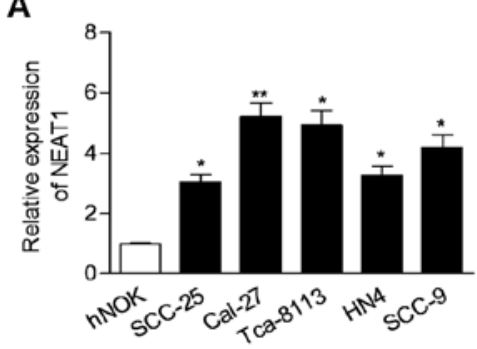

D
B

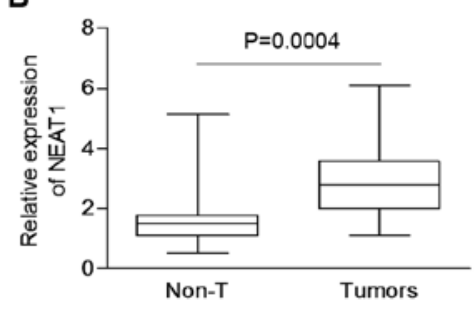

E
C

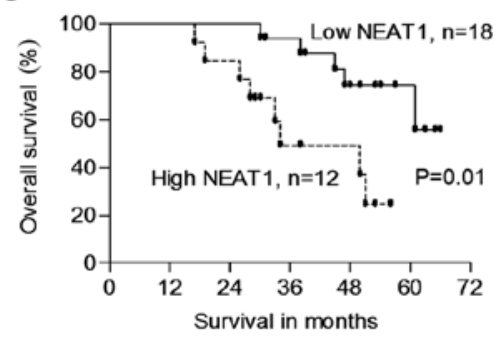

F

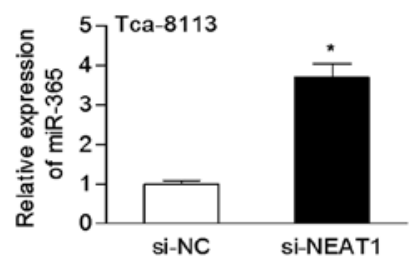

G

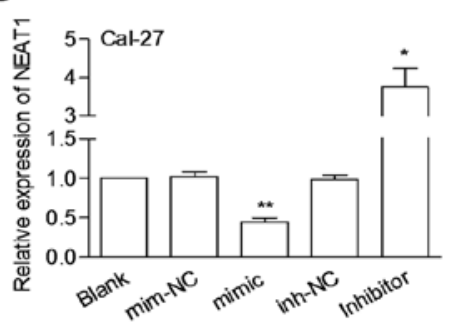

$\mathbf{J}$

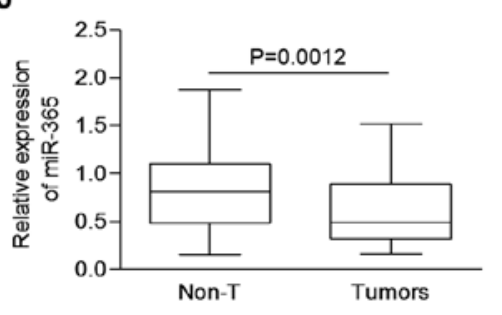

H

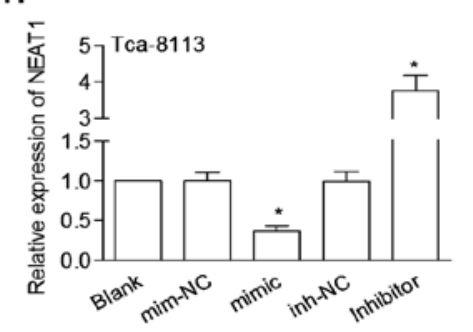

K

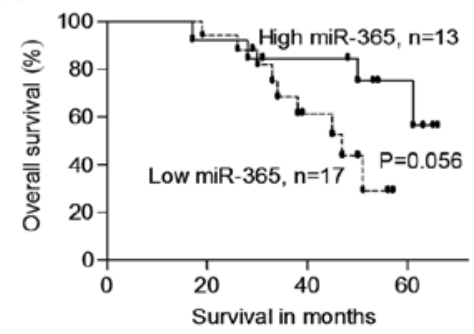

I

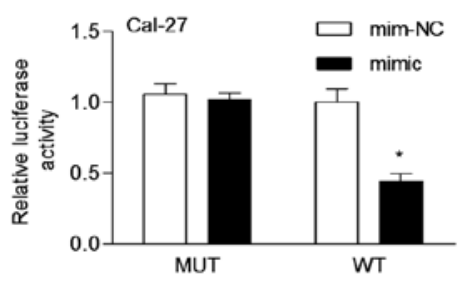

L

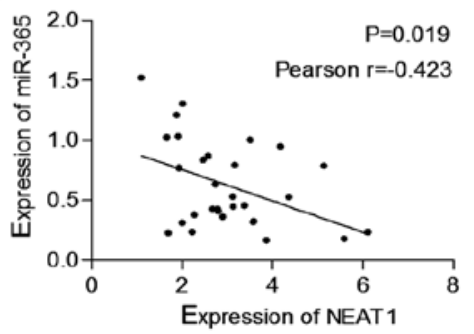

Figure 1. NEAT1 is negatively correlated with miR-365. (A and B) The expression of NEAT1 in OSCC (A) cells and (B) tissues was determined by qPCR. (C) Low expression of NEAT1 indicated a better prognosis of patients. (D) Binding site was predicted between NEAT1 and miR-365. (E and F) The expression of miR-365 was increased in (E) Cal-27 and (F) Tca-8113 cells transfected with si-NEAT1. (G and H) The expression of NEAT1 was decreased by the miR-365 mimic and increased by the miR-365 inhibitor in (G) Cal-27 and (H) Tca-8113 cells. (I) Luciferase activity of reporters containing the NEAT1-WT or NEAT1-MUT sequence in Cal-27 cells transfected with the miR-365 mimic or mim-NC. (J) miR-365 was significantly downregulated in OSCC tissues. (K) Low expression of miR-365 was associated with poor survival of patients. (L) The expression of NEAT1 was inversely correlated with miR-365 in tumors. ${ }^{*} \mathrm{P}<0.05,{ }^{* *} \mathrm{P}<0.01$. OSCC, oral squamous cell carcinoma; qPCR, quantitative real-time PCR; si-NC, negative control siRNA; mim-NC, mimic negative control; inh-NC, inhibitor negative control; si-NEAT1, specifically targeting NEAT1; WT, wild-type; MUT, mutant.

Elevated NEAT1 is correlated with aggressive tumor phenotypes and poor prognosis. OSCC cases were classified into different subgoups such assex (male vs. female) and TNM stage (I/II vs. III/IV). We determined that the expression levels of NEAT1 were significantly increased in cases with lymph node metastasis $(\mathrm{P}=0.009)$ and higher clinical stage $(\mathrm{P}=0.018$; Table I), respectively. The median level of NEAT1 in tumors was used as a cut-off value to divide cases into two groups. Patients with high NEAT1 expression had poor survival when compared to patients with low NEAT1 expression $(\mathrm{P}=0.01$; Fig. 1C).

NEAT1 negatively regulates miR-365 in OSCC. The exact function and underlying mechanism of NEAT1 in OSCC warranted further investigation. Using starBase 2.0 and RegRNA2.0, miR-365 was determined to potentially bind to
NEAT1 (Fig. 1D), implying a possible interaction between miR-365 and NEAT1. Cal-27 and Tca-8113 cells, which express a relatively high level of NEAT1, were used for further analysis. We determined that the levels of miR-365 were significantly increased by si-NEAT1 transfection in both Cal-27 and Tca-8113 cells (Fig. 1E and F). The expression of NEAT1 was downregulated by miR-365 mimic, while it was upregulated by the miR-365 inhibitor (Fig. $1 \mathrm{G}$ and $\mathrm{H}$ ). Co-transfection of miR-365 and NEAT1-WT significantly decreased the luciferase activity (Fig. 1I).

miR-365 was significantly downregulated in OSCC tissues compared to non-tumor tissues $(0.622 \pm 0.364$ vs. $0.819 \pm 0.428$, $\mathrm{P}=0.0012$; Fig. 1J). miR-365 was significantly downregulated in tumors of advanced stage $(\mathrm{P}=0.039$, Table I). Cases were grouped in a low or high group according to the median level of miR-365. Patients with low expression of miR-365 
A

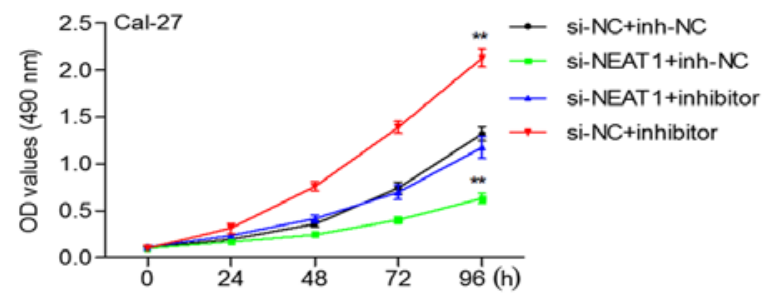

B
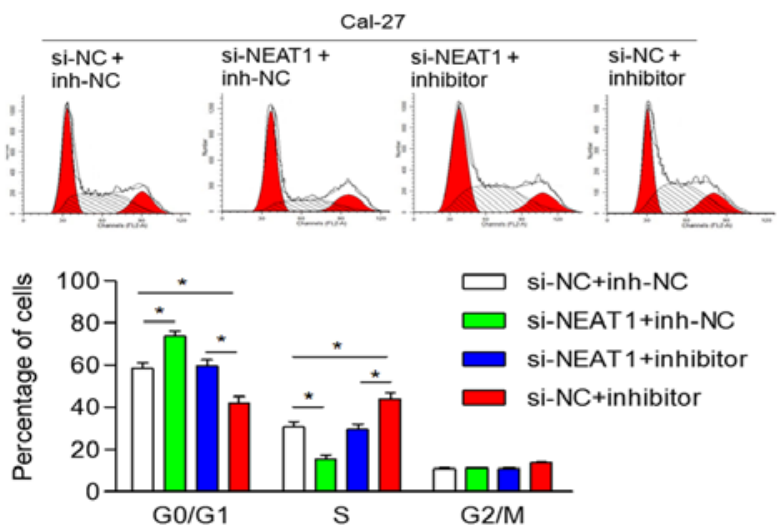

C
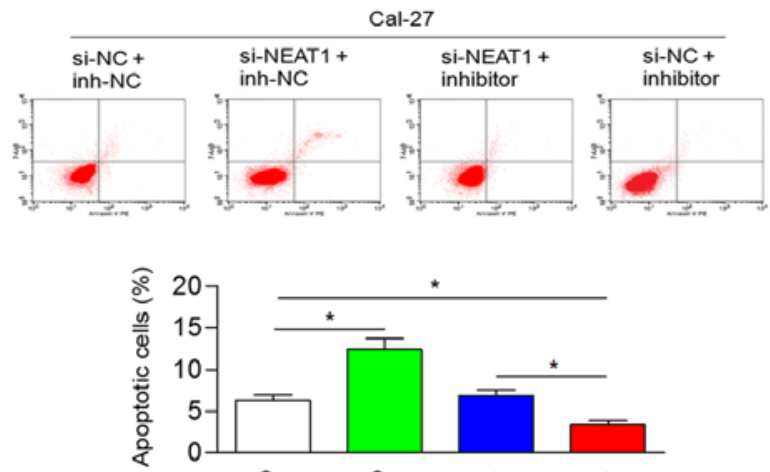

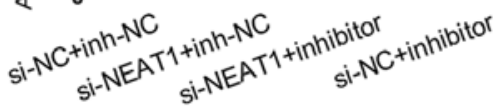

D
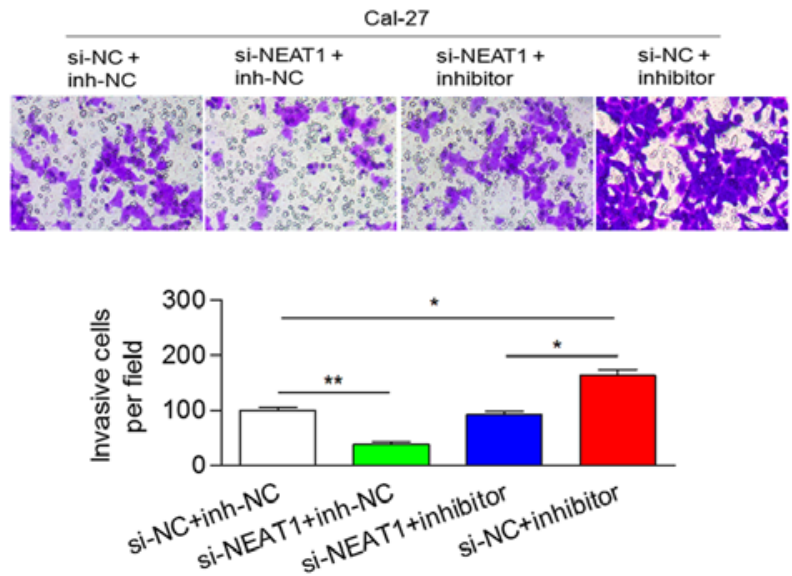
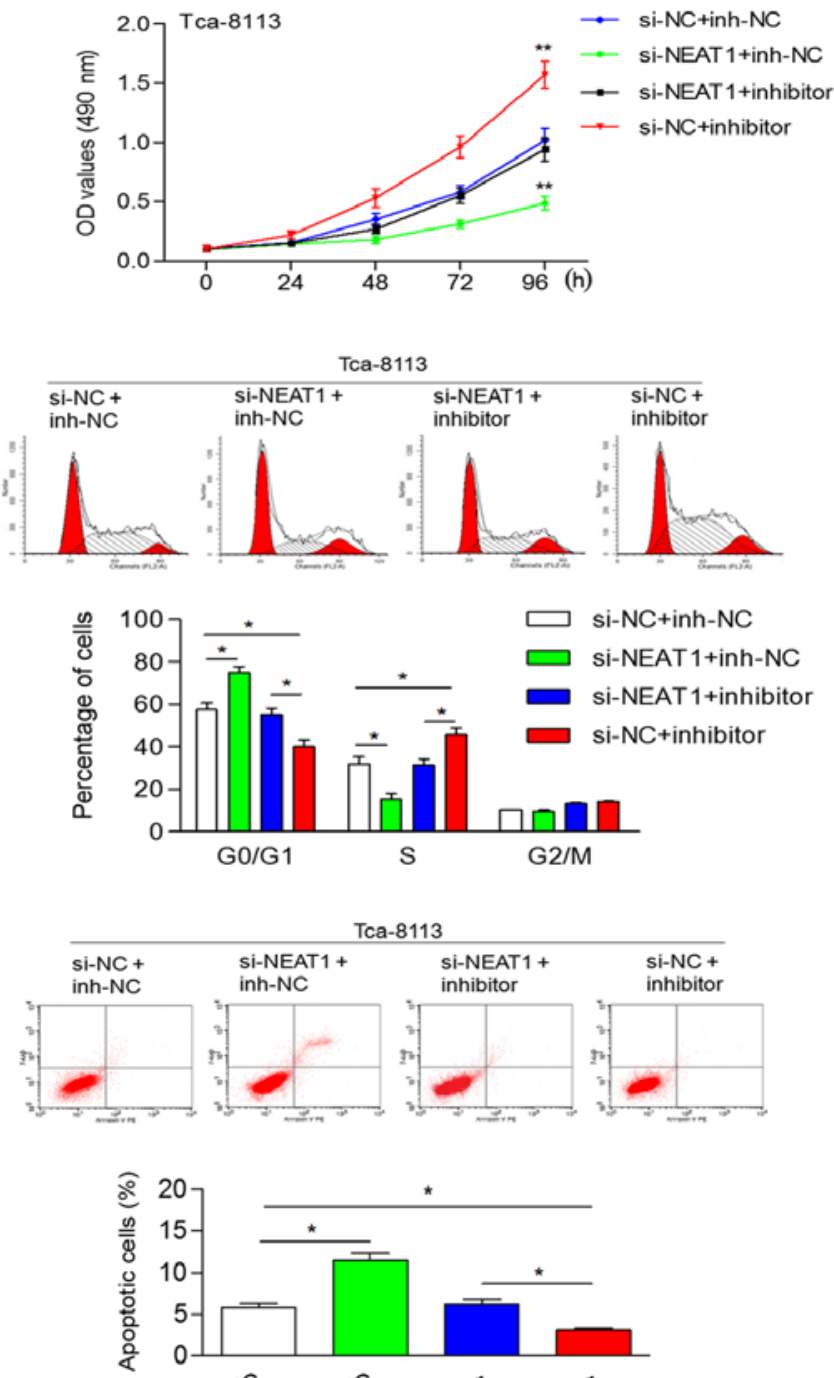

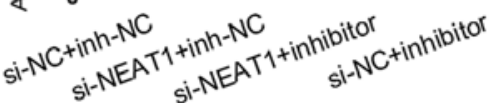
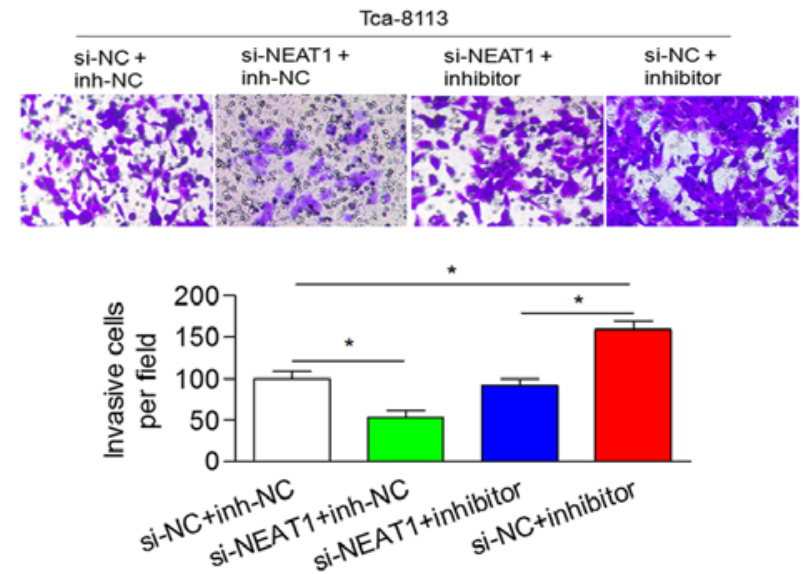

Figure 2. miR-365 inhibitor attenuates the suppressive effect of knockdown of NEAT1 on cell growth and motility. (A) An MTT assay was conducted to evaluate the effect of the miR-365 inhibitor and si-NEAT1 on cell proliferation. (B) Knockdown of NEAT1 induced an increase in the percentage of cells at the G0/G1 phase and a decrease of cells at the $\mathrm{S}$ phase, a phenomenon that could be reversed by the miR-365 inhibitor. (C) Knockdown of NEAT1 contributed to cell apoptosis, whereas the miR-365 inhibitor had the opposite effect on cell apoptosis. (D) The number of invasive cells was increased by the miR-365 inhibitor, while it was reduced by si-NEAT1. ${ }^{*} \mathrm{P}<0.05,{ }^{* * *} \mathrm{P}<0.01$. si-NC, negative control siRNA; inh-NC, inhibitor negative control; si-NEAT1, specifically targeting NEAT1.

appeared to have poor prognosis but without statistical significance $(\mathrm{P}=0.056$; Fig. $1 \mathrm{~K})$. In addition, NEAT1 expression was negatively correlated with the expression of miR-365 in tumors $(\mathrm{P}=0.019$, Pearson $\mathrm{r}=-0.423$; Fig. 1L). These findings 
A

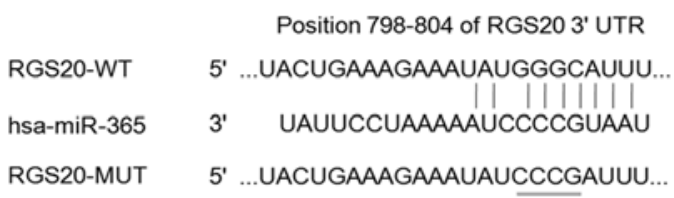

C

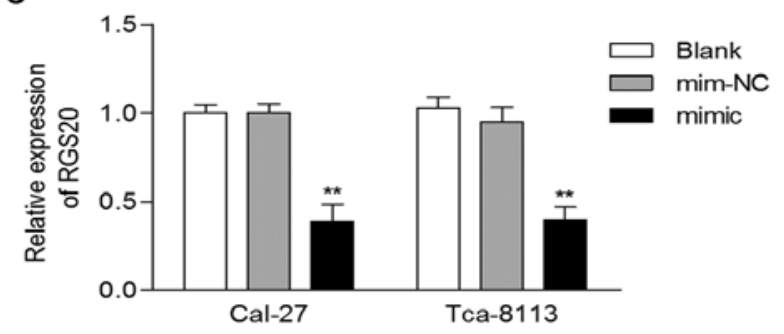

E

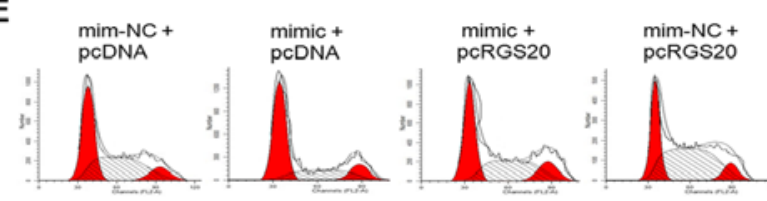

F

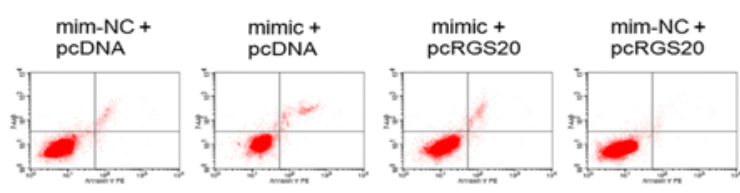

G

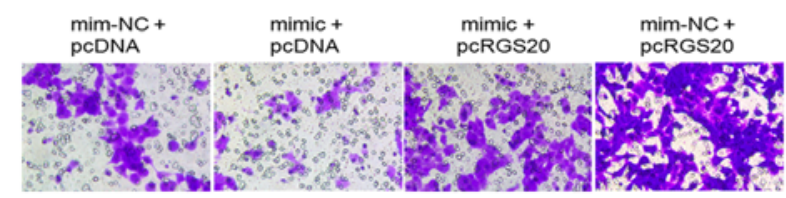

B

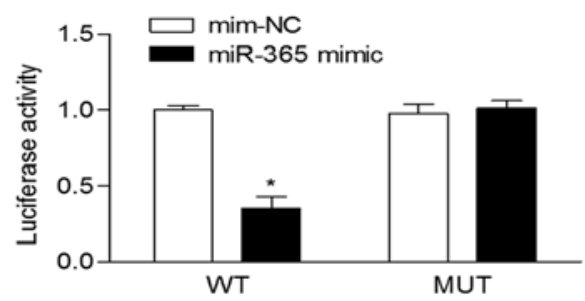

D
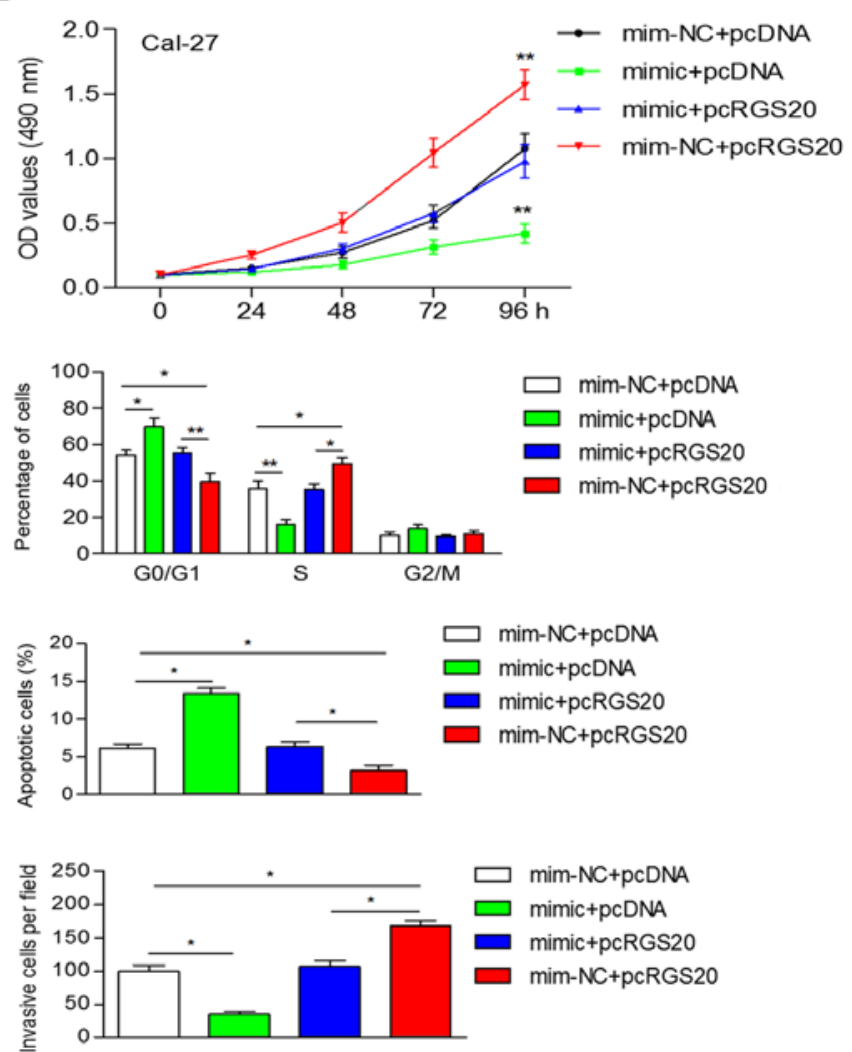

Figure 3. RGS20 was a functional target of miR-365. (A) Representative binding sites of miR-365 on the 3'-UTR of NEAT1 mRNA. (B) Luciferase reporter assay for RGS20 mRNA 3'-UTR following miR-365 ectopic expression. (C) The mRNA levels of RGS20 were inhibited by miR-365 mimic in OSCC cells. (D) Cell proliferation was determined in these cells. (E) Ectopic expression of miR-365 led to a sharp decrease of cells at the S phase and overexpression of RGS20 (lacking the 3'-UTR) promoted cell cycle transition from the G0/G1 to the S phase. (F) miR-365 increased the number of apoptotic cells, which could be inhibited by RGS20 overexpression. (G) The miR-365 mimic abolished the increase of invasive cells induced by RGS20. ${ }^{*} \mathrm{P}<0.05$, ${ }^{* *} \mathrm{P}<0.01$. UTR, untranslated region; OSCC, oral squamous cell carcinoma; WT, wild-type; MUT, mutant; mim-NC, mimic negative control.

indicated that an interaction between NEAT1 and miR-365 may be involved in the development of OSCC.

Inhibition of miR-365 attenuates the NEATl knockdown-induced inhibition of cellular processes. To explore the effect of NEAT1 knockdown and miR-365 inhibition on cellular processes, we transfected OSCC cells with si-NEAT1 (or si-NC) and miR-365 inhibitor (or inh-NC). Knockdown of NEAT1 significantly reduced cell proliferation and abrogated the miR-365 inhibitor-induced increase of the cell proliferation rate (Fig. 2A). Flow cytometric analysis revealed that si-NEAT1 induced an increase in the percentage of cells at the G0/G1 phase and a reduction in the percentage of cells at the $\mathrm{S}$ phase, while the miR-365 inhibitor had an opposite effect on cell cycle distribution (Fig. 2B). Knockdown of NEAT1 induced cell apoptosis and abolished the miR-365 inhibitor-induced decrease of apoptotic cells (Fig. 2C). In addition, knockdown of NEAT1 could inhibit the invasive ability of cells, which was promoted by the miR-365 inhibitor (Fig. 2D). These results revealed that NEAT1 contributed to cell proliferation and invasion by negatively-mediated miR-365.

$R G S 20$ is a target of $m i R-365$. Numerous studies have revealed that lncRNAs could competitively suppress miRNAs by acting as ceRNAs, and ultimately regulate the expression of protein-coding genes. Thus, we searched for candidate genes of miR-365 using TargetScan, microRNA, miRDB and TargetMiner. Among the predicted targets, in the present study we focused on RGS20, considering the involvement of RGS20 in human cancers. In addition, by analyzing datasets from Oncomine, we determined that the mRNA levels of RGS20 were significantly upregulated in tongue squamous cell carcinoma (data not shown). All four bioinformatics tools revealed that the 3'-UTR of RGS20 mRNA has a potential binding site of miR-365 (Fig. 3A). Furthermore, a luciferase reporter assay revealed that the miR-365 mimic significantly reduced the luciferase activity of the wild-type 3'-UTR of RGS20 (Fig. 3B). 
A

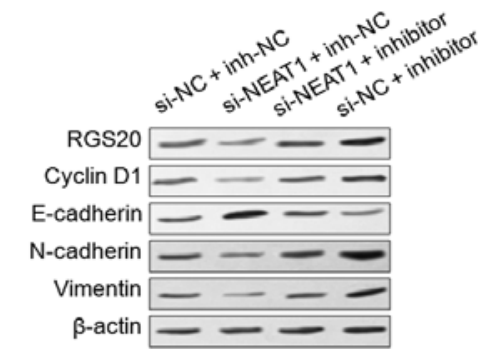

C
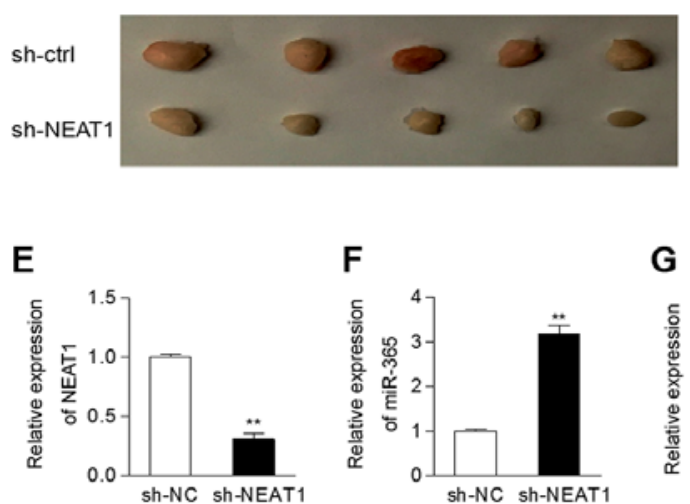

F

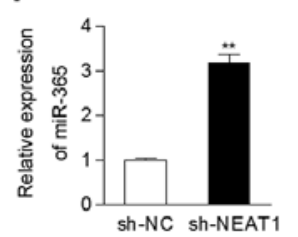

B

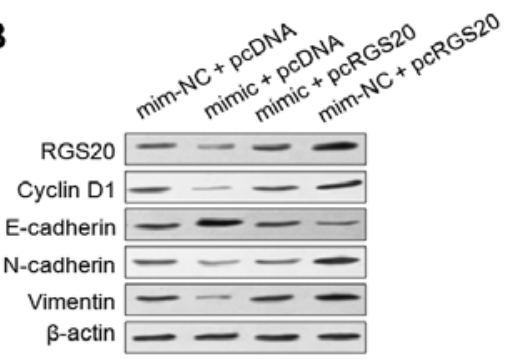

D

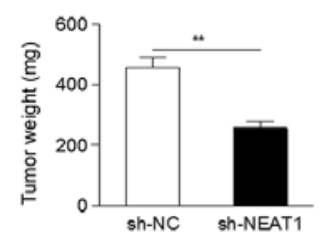

G

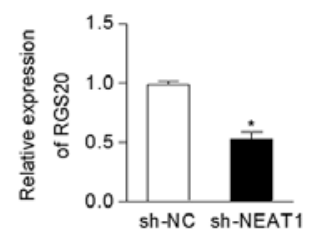

H

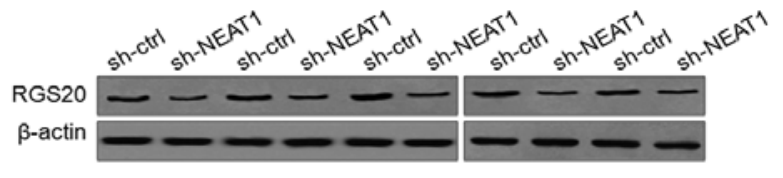

Figure 4. Dysregulationof the NEAT1/miR-365/RGS20 axis is involved in EMT and tumor growth. (A and B) The protein levels of RGS20, cyclin D1, E-cadherin, N-cadherin and vimentin in Cal-27 cells following (A) knockdown of NEAT1 and/or inhibition of miR-365, as well as in (B) cells transfected with mimic (or mim-NC) and pcRGS20 (or pcDNA). (C and D) Knockdown of NEAT1 led to a marked reduction of (C) the tumor volume and (D) tumor weight. The expression of (E) NEAT1, (F) miR-365 and (G) RGS20 was determined in mouse tumors. (H) The protein levels of RGS20 of mouse tumors were determined by western blot analysis. ${ }^{*} \mathrm{P}<0.05,{ }^{* *} \mathrm{P}<0.01$. EMT, epithelial-mesenchymal transition; mim-NC, mimic negative control; si-NC, negative control siRNA; inh-NC, inhibitor negative control; si-NEAT1, specifically targeting NEAT1.

In addition, overexpression of miR-365 significantly decreased the mRNA expression of RGS20 in OSCC cells (Fig. 3C).

RGS20 is a functional target of $m i R-365$. To ascertain whether miR-365 performs its suppressive function through downregulation of RGS20, Cal-27 cells were co-transfected with pcRGS20 or the 3'-UTR (or pcDNA) and with miR-365 mimic (or mim-NC). Cell proliferation was stimulated by overexpression of RGS20 and inhibited by the miR-365 mimic (Fig. 3D). Cell cycle analysis revealed that pcRGS20 transfected cells displayed a higher frequency of cells at the $\mathrm{S}$ phase and a lower frequency of cells at the G1 phase and ectopic expression of RGS20 reversed the miR-365-induced accumulation of G0/G1 phase cells (Fig. 3E). The apoptosis of Cal-27 cells was increased by the miR-365 mimic, while it was promoted decreased by the overexpression of RGS20 (Fig. 3F). Overexpression of RGS20 increased cell invasion (Fig. 3G), which was similar to the effect of the miR-365 inhibitor. RGS20 overexpression also significantly attenuated miR-365-induced inhibition on cellular invasion (Fig. 3G). These data indicated that miR-365 performs its tumor-suppressive function by regulating RGS20.

Dysregulation of the NEAT1/miR-365/RGS20 axis is involved in epithelial-mesenchymal transition (EMT) and tumor growth. Western blot analysis was performed to evaluate the effect of NEAT1/miR-365/RGS20 on the protein expression of cell cycle- and EMT-related markers. As shown in Fig. 4A, the protein expression of RGS20 was decreased by knockdown of NEAT1, while it was increased by the miR-365 inhibitor.
Inhibition of NEAT1 abolished the miR-365 inhibitor-induced upregulation of RGS20 (Fig. 4A). Downregulation of NEAT1 led to an increase of E-cadherin and a reduction of cyclin D1, $\mathrm{N}$-cadherin and vimentin, a phenomenon that could be reversed by the miR-365 inhibitor (Fig. 4A). Subsequently, the protein level of RGS20 could be inhibited by the miR-365 mimic (Fig. 4B), which was consistent with previous data shown in Fig. 3C. Restoration of RGS20 promoted the protein expression of cyclin D1, N-cadherin and vimentin, but suppressed the protein expression of E-cadherin (Fig. 4B).

To ascertain whether knockdown of NEAT1 inhibits tumor growth in vivo, Cal-27 cells (expressing sh-NEAT1or sh-ctrl) were injected into the flanks of nude mice. The results indicated that the tumor volumes and weights formed by the sh-NEAT1 cells were markedly lower than those formed by the sh-ctrl cells (Fig. 4C and D). In addition, the tumors of sh-NEAT1-treated mice had a significantly low level of NEAT1, increased expression of miR-365, and downregulation of RGS20 (Fig. 4E-G). Furthermore, the protein levels of RGS20 were decreased in mouse tumors transfected with sh-NEAT1 compared to the control group (Fig. 4H).

\section{Discussion}

Numerous studies have shown that lncRNAs function as oncogenes or tumor-suppressor genes to regulate carcinogenesis, and that they can be used as diagnostic or prognostic markers (20). In the present study, the expression of NEAT1 was markedly increased in OSCC cells and tissues, and upregulation of NEAT1 was correlated with advanced stage and unfavorable 
prognosis of OSCC patients. Similarly, high expression of NEAT1 was associated with metastasis and vaso-invasion in hepatocellular carcinoma (21). High NEAT1 was closely related to larger tumor size and independently associated with risk of death in glioma (22), as well as clinical pathologic grade in bladder cancer (23). Our results revealed the oncogenic role of NEAT1 in the development of OSCC. Certainly, further analysis based on a larger number of cases would provide more knowledge on the clinical relevance of NEAT1 in OSCC.

Functionally, knockdown of NEAT1 exerted a tumorsuppressive effect by inhibiting cell proliferation, cell cycle progression, and invasion in vitro and tumorigenesis in vivo, which was consistent with previous studies (10,23-25). NEAT1 could negatively regulate the expression of miR-365. miR-365 inhibition abrogated the inhibitory effect of NEAT1 knockdown. Several other miRNAs, including miR-377, miR-335, miR-107, miR-98 and miR-506, were identified to interact with NEAT1 in different types of cancers $(12,14,26-28)$, suggesting that NEAT1 plays an oncogenic role in different types of cancer through the regulation of different miRNAs.

Our findings revealed that miR-365 suppressed cell proliferation and invasion and expanded on the knowledge of miR-365 as a tumor suppressor in OSCC. The inhibitory effect of miR-365 on tumorigenesis has also been reported in several studies (17-19,29). However, miR-365 displayed the opposite effect in cutaneous tumors by facilitating tumor growth (30). Thus, miR-365 exerts a tumor-suppressive or oncogenic function depending on its target genes. In the present study, RGS20 was identified as a direct target of miR-365 and overexpression of RGS20 impaired the miR-365-induced inhibition of cell growth and invasion. In addition, cyclin D1, CDC25A, WNT5A and ADAM10 were identified as targets of miR-365 and were correlated with miR-365-mediated cell growth and metastasis $(19,29,31)$.

RGS20 was first reported to be overexpressed in metastatic melanomas (32). High expression of RGS20 indicated the progression and poor survival of triple-negative breast cancer (33). RGS20 facilitated cell aggregation, invasion and the expression of vimentin, but decreased the expression of E-cadherin (34). By gain-of-function approaches, we revealed similar results. RGS20 increased cell viability, motility and protein expression of cyclin D1, N-cadherin but decreased the protein level of E-cadherin, suggesting the oncogenic function of RGS20 in OSCC. The protein level of RGS20 was regulated by NEAT1/miR-365, suggesting that NEAT1 acted as a ceRNA of miR-365 and enhanced the expression of RGS20. Moreover, cell cycle- and EMT-related indicators were regulated by the NEAT1/miR-365/RGS20 pathway, supporting the regulatory effect of the NEAT1/miR-365/RGS20 axis on cell growth and metastasis in vitro. A previous study also demonstrated that NEAT1 is a regulator of EMT-related proteins in gastric cancer (35). Our findings revealed that RGS20, a direct target of miR-365, could mediate the biological effects that NEAT1 exerted.

In conclusion, we determined that upregulated NEAT1 was correlated with an aggressive tumor phenotype and an adverse prognosis in OSCC. NEAT1 promoted OSCC cell proliferation, cell cycle progression and invasion through the miR-365/RGS20 axis. These data provide new insights into the regulatory function of NEAT1/miR-365/RGS20 in the development of oral malignancy.

\section{Acknowledgements}

Not applicable.

\section{Funding}

No funding was received.

\section{Availability of data and materials}

Not applicable.

\section{Authors' contributions}

GH conceived and designed the experiments. GH and XLW performed the experiments. $\mathrm{XH}$ analyzed the data. GH and $\mathrm{XH}$ wrote the manuscript. All authors contributed toward data analysis, drafting and critically revising the paper, gave final approval of the version to be published.

\section{Ethics approval and consent to participate}

The present study was approved by the Research Ethics Committee of the General Hospital of Benxi Iron and Steel Co., Ltd., and written informed consents from patients were signed before surgery. All animal procedures were in line with the guidelines of the Laboratory Animal Centre and were approved by the Ethics Committee of the General Hospital of Benxi Iron and Steel Co., Ltd.

\section{Consent for publication}

Not applicable.

\section{Competing interests}

The authors declare that they have no competing interests.

\section{References}

1. Warnakulasuriya S: Global epidemiology of oral and oropharyngeal cancer. Oral Oncol 45: 309-316, 2009.

2. Hoffman HT, Porter K, Karnell LH, Cooper JS, Weber RS, Langer CJ, Ang KK, Gay G, Stewart A and Robinson RA: Laryngeal cancer in the United States: Changes in demographics, patterns of care, and survival. Laryngoscope 116: 1-13, 2006.

3. Bayoumi AS, Sayed A, Broskova Z, Teoh JP, Wilson J, Su H, Tang YL and Kim IM: Crosstalk between long noncoding RNAs and microRNAs in health and disease. Int J Mol Sci 17: 356, 2016.

4. Schmitt AM and Chang HY: Long noncoding RNAs in cancer pathways. Cancer Cell 29: 452-463, 2016.

5. Gregory RI and Shiekhattar R: MicroRNA biogenesis and cancer. Cancer Res 65: 3509-3512, 2005.

6. Li J, Tian H, Yang J and Gong Z: Long noncoding RNAs regulate cell growth, proliferation, and apoptosis. DNA Cell Biol 35: 459-470, 2016 .

7. Shenouda SK and Alahari SK: MicroRNA function in cancer: Oncogene or a tumor suppressor? Cancer Metastasis Rev 28: 369-378, 2009.

8. Li Y, Li Y, Chen W, He F, Tan Z, Zheng J, Wang W, Zhao Q and Li J: NEAT expression is associated with tumor recurrence and unfavorable prognosis in colorectal cancer. Oncotarget 6: 27641-27650, 2015.

9. Wu Y, Yang L, Zhao J, Li C, Nie J, Liu F, Zhuo C, Zheng Y, Li B Wang $\mathrm{Z}$ and $\mathrm{Xu} \mathrm{Y}$ : Nuclear-enriched abundant transcript 1 as a diagnostic and prognostic biomarker in colorectal cancer. Mol Cancer 14: 191, 2015. 
10. Chen X, Kong J, Ma Z, Gao S and Feng X: Up regulation of the long non-coding RNA NEAT1 promotes esophageal squamous cell carcinoma cell progression and correlates with poor prognosis. Am J Cancer Res 5: 2808-2815, 2015.

11. Chakravarty D, Sboner A, Nair SS, Giannopoulou E, Li R, Hennig S, Mosquera JM, Pauwels J, Park K, Kossai M, et al: The oestrogen receptor alpha-regulated lncRNA NEAT1 is a critical modulator of prostate cancer. Nat Commun 5: 5383, 2014.

12. Sun C, Li S, Zhang F, Xi Y, Wang L, Bi Y and Li D: Long non-coding RNA NEAT1 promotes non-small cell lung cancer progression through regulation of miR-377-3p-E2F3 pathway. Oncotarget 7: 51784-51814, 2016.

13. Lo PK, Zhang Y, Wolfson B, Gernapudi R, Yao Y, Duru N and Zhou Q: Dysregulation of the BRCA1/long non-coding RNA NEAT1 signaling axis contributes to breast tumorigenesis. Oncotarget 7: 65067-65089, 2016.

14. Cao J, Zhang Y, Yang J, He S, Li M, Yan S, Chen Y, Qu C and $\mathrm{Xu}$ L: NEAT1 regulates pancreatic cancer cell growth, invasion and migration though mircroRNA-335-5p/c-met axis. Am J Cancer Res 6: 2361-2374, 2016.

15. Yang X, Xiao Z, Du X, Huang L and Du G: Silencing of the long non-coding RNA NEAT1 suppresses glioma stem-like properties through modulation of the miR-107/CDK6 pathway. Oncol Rep 37: 555-562, 2017.

16. Qian K, Liu G, Tang Z, Hu Y, Fang Y, Chen Z and Xu X: The long non-coding RNA NEAT1 interacted with miR-101 modulates breas cancer growth by targeting EZH2. Arch Biochem Biophys 615: 1-9, 2017.

17. Nie J, Liu L, Zheng W, Chen L, Wu X, Xu Y, Du X and Han W: microRNA-365, down-regulated in colon cancer, inhibits cell cycle progression and promotes apoptosis of colon cancer cells by probably targeting cyclin D1 and Bcl-2. Carcinogenesis 33 : 220-225, 2012

18. Kang SM, Lee HJ and Cho JY: MicroRNA-365 regulates NKX2-1, a key mediator of lung cancer. Cancer Lett 335: 487-494, 2013.

19. Guo SL, Ye H, Teng Y, Wang YL, Yang G, Li XB, Zhang C, Yang X, Yang ZZ and Yang X: Akt-p53-miR-365-cyclin D1/cdc25A axis contributes to gastric tumorigenesis induced by PTEN deficiency. Nat Commun 4: 2544, 2013.

20. Chandra Gupta S and Nandan Tripathi Y: Potential of long non-coding RNAs in cancer patients: From biomarkers to therapeutic targets. Int J Cancer 140: 1955-1967, 2017.

21. Guo S, Chen W, Luo Y, Ren F, Zhong T, Rong M, Dang Y, Feng Z and Chen G: Clinical implication of long non-coding RNA NEAT1 expression in hepatocellular carcinoma patients. Int J Clin Exp Pathol 8: 5395-5402, 2015

22. He C, Jiang B, Ma J and Li Q: Aberrant NEAT1 expression is associated with clinical outcome in high grade glioma patients. APMIS 124: 169-174, 2016.
23. Gong W, Zheng J, Liu X, Ma J, Liu Y and Xue Y: Knockdown of NEAT1 restrained the malignant progression of glioma stem cells by activating microRNA let-7e. Oncotarget 7: 62208-62223, 2016.

24. Li JH, Zhang SQ, Qiu XG, Zhang SJ, Zheng SH and Zhang DH: Long non-coding RNA NEAT1 promotes malignant progression of thyroid carcinoma by regulating miRNA-214. Int J Oncol 50: 708-716, 2017.

25. Jen J, Tang YA, Lu YH, Lin CC, Lai WW and Wang YC: Oct4 transcriptionally regulates the expression of long non-coding RNAs NEAT1 and MALAT1 to promote lung cancer progression. Mol Cancer 16: 104, 2017.

26. Wang P, Wu T, Zhou H, Jin Q, He G, Yu H, Xuan L, Wang X, Tian L, Sun Y, et al: Long noncoding RNA NEAT1 promotes laryngeal squamous cell cancer through regulating miR-107/CDK6 pathway. J Exp Clin Cancer Res 35: 22, 2016

27. Jiang $P$, Wu X, Wang $X$, Huang $W$ and Feng Q: NEAT1 upregulates EGCG-induced CTR1 to enhance cisplatin sensitivity in lung cancer cells. Oncotarget 7: 43337-43351, 2016.

28. Huang B, Liu C, Wu Q, Zhang J, Min Q, Sheng T, Wang X and Zou Y: Long non-coding RNA NEAT1 facilitates pancreatic cancer progression through negative modulation of miR-506-3p. Biochem Biophys Res Commun 482: 828-834, 2017.

29. Wang Y, Xu C, Wang Y and Zhang X: MicroRNA-365 inhibits ovarian cancer progression by targeting Wnt5a. Am J Cancer Res 7: 1096-1106, 2017.

30. Zhou M, Zhou L, Zheng L, Guo L, Wang Y, Liu H, Ou C and Ding Z: miR-365 promotes cutaneous squamous cell carcinoma (CSCC) through targeting nuclear factor I/B (NFIB). PLoS One 9: e100620, 2014.

31. Liu Y, Zhang W, Liu S, Liu K, Ji B and Wang Y: miR-365 targets ADAM10 and suppresses the cell growth and metastasis of hepatocellular carcinoma. Oncol Rep 37: 1857-1864, 2017.

32. Riker AI, Enkemann SA, Fodstad O, Liu S, Ren S, Morris C, Xi Y, Howell P, Metge B, Samant RS, et al: The gene expression profiles of primary and metastatic melanoma yields a transition point of tumor progression and metastasis. BMC Med Genomics 1: 13, 2008.

33. Li Q, Jin W, Cai Y, Yang F, Chen E, Ye D, Wang Q and Guan X: Regulator of $\mathrm{G}$ protein signaling 20 correlates with clinicopathological features and prognosis in triple-negative breast cancer. Biochem Biophys Res Commun 485: 693-697, 2017.

34. Yang L, Lee MM, Leung MM and Wong YH: Regulator of G protein signaling 20 enhances cancer cell aggregation, migration, invasion and adhesion. Cell Signal 28: 1663-1672, 2016.

35. Fu JW, Kong Y and Sun X: Long noncoding RNA NEAT1 is an unfavorable prognostic factor and regulates migration and invasion in gastric cancer. J Cancer Res Clin Oncol 142: 1571-1579, 2016. 